\title{
BGO Track Reconstruction with DAMPE
}

\author{
Shi-Jun Lei* \\ Key Laboratory of Dark Matter and Space Astronomy, Purple Mountain Observatory, Chinese \\ Academy of Sciences, Nanjing 210008, China \\ E-mail: sjlei@pmo.ac.cn

\section{Wei Jiang} \\ Key Laboratory of Dark Matter and Space Astronomy, Purple Mountain Observatory, Chinese \\ Academy of Sciences, Nanjing 210008, China \\ E-mail: jiangwei@pmo.ac.cn
}

\section{On behalf of the DAMPE collaboration}

The BGO calorimeter (BGO) on board the DArk Matter Particle Explorer (DAMPE) is composed of 308 BGO scintillation crystals hodoscopically arranged in 14 layers to measure the threedimensional shower profile of particles. In addition to the measurement of particle energy and differentiating between electromagnetic and hadronic showers, the BGO image is also used to measure the direction of the incident particle despite its moderate resolution. Making use of the entire shower image in the BGO, the BGO shower axis reconstructed is less effected by the backsplash effect and secondary particles, in good complement to the STK track especially for high energy events. The BGO track reconstructed is used as a seed for the STK track reconstruction as well as to identify the STK track best associate with the incident particle. We introduce in this proceeding an improved BGO track reconstruction algorithm that provides results of higher accuracy as demonstrated using both flight data and simulation data.

36th International Cosmic Ray Conference -ICRC2019-

July 24th - August 1st, 2019

Madison, WI, U.S.A.

\footnotetext{
* Speaker.

${ }^{\dagger}$ for collaboration list see PoS(ICRC2019)1177.
} 


\section{Introduction}

The Dark Matter Particle Explorer (DAMPE) is a space-born high-energy cosmic-ray and Gamma-ray detector successfully launched on December 17, 2015 [1, 2]. With coordinated detection of the four sub-detectors, namely, the plastic scintillation detector (PSD), the silicon-tungsten Tracker (STK), the BGO calorimeter (BGO), and the neutron detector, DAMPE is able to measure the charge, direction, and energy of an incident particle, as well as to discriminate particles of different types, in a very wide energy range.

Measurement of the direction of incident particles via track reconstruction is one of the key function of DAMPE. Not only the direction measurement is critical in dressing various science problems of great significance, such as the gamma-ray source identification [3], the space structure of dark matter [4, 5, 6], the origin and propagation of cosmic-rays [7], the track reconstruction also acts as a precondition in charge measurement [8] and Gamma-ray selection [10]. During the period of on-orbit operation up to now, significant progress in direction measurement has been made given our better understanding of the DAMPE detector based on accumulating flight data and simulation data. We introduce in this proceeding the evolution of the BGO track reconstruction, focusing on the new algorithm that provides measurements of significantly improved accuracy, as validated using both the flight and simulation data.

\section{Overview of DAMPE track reconstruction}

The main instrument for direction measurement is the STK, which is able to provide a resolution of $\sim 0.1^{\circ}$ (for $100 \mathrm{GeV}$ electrons/gamma-rays) detection channels in total [9]. However, as shown in Fig. 1, the STK track reconstruction could be complicated by the backsplash and/or secondary particles, which may generate spurious track(s) in addition to that associated with the incident particle (hereby, the real track). Generally speaking, the situation deteriorates for particles of even higher energy, and it could be very challenging to identify the real track desired among dozens of tracks reconstructed, not to mention that it is also very time consuming to reconstruct all of them from hundreds of STK hits.

The BGO calorimeter, composed of 308 BGO scintillation crystals hodoscopically arranged in 14 layers, is mainly used for energy measurement and electron/hadron discrimination. Despite its limited spatial resolution (a few degrees) due to the much larger dimension of each BGO bar $(25 \mathrm{~mm} \times 25 \mathrm{~mm} \times 600 \mathrm{~mm}$ ), the BGO detector can also be used for the track reconstruction. Except a small fraction of minimum ionizing particles (MIPs), which but already have the most distinct tracks, most events shower in the BGO given the 32 radiation lengths of the BGO detector from top to bottom. For most events, the energy deposition patten recorded by the BGO is an accumulating imaging of the shower not so sensitive to single backsplash or secondary particle, and the BGO shower axis reconstructed is a fairly reliable estimation of the direction of the incident particle within the uncertainty. Actually, the shower pattern is in general more extended and clear-cut for events of higher energy to provide even more accurate track reconstruction, in very good complement with the STK track. Also, comparing to the STK track reconstruction applying a Kalman filter method, the BGO track reconstruction based on linear fit is of much slighter computational resource consumption. The BGO track, less precise but easily reconstructed and reliable, is used 
to provide a rough guidance for the STK track reconstruction, as well as help to identify the track best associated with incident particle among several candidates, so as to provide the best overall direction measurement of DAMPE. In the extreme case when the STK track reconstruction is not possible, the BGO track could be the only way to provide direction information.

$\mathrm{Y}$ [No. 32: 49.132GeV]

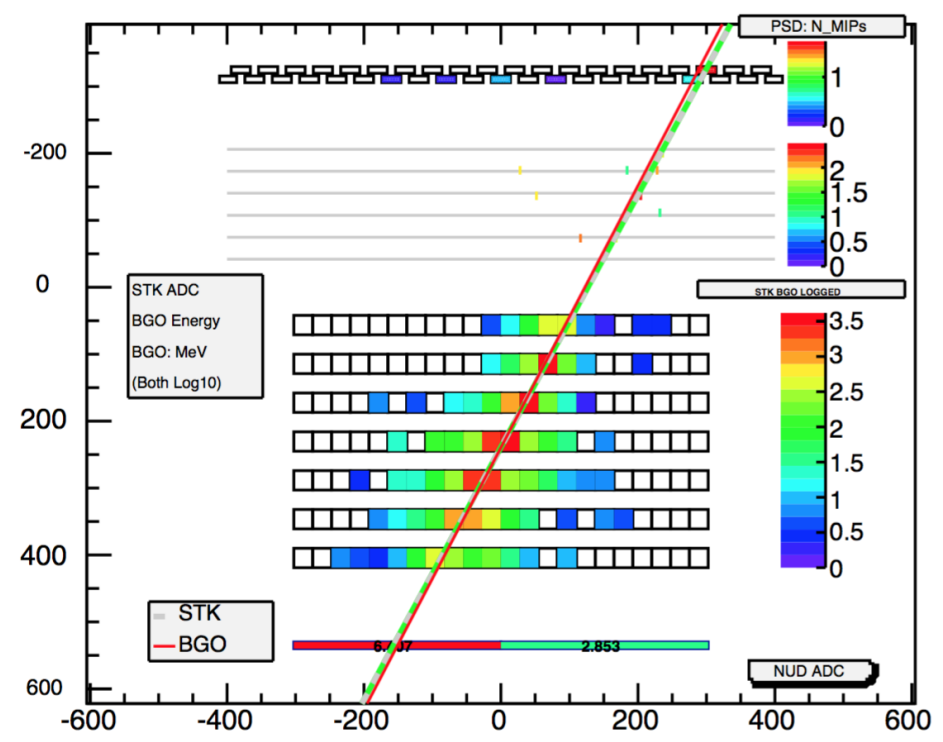

Figure 1: Tracks reconstructed for a typical proton event. With the help of the BGO track (red solid line) reconstructed as the shower axis of the whole BGO shower image, the correct STK track (green dash line) is reconstructed despite several contaminating hits due to backsplash/secondary particles. The STK track is used to determined the correct PSD channels penetrated by the incident particle ultimately identified as a proton.

\section{BGO track reconstruction: a first attempt}

Considering the beneficial role played by BGO track, the BGO track reconstruction is included in the data procession since the very beginning. As a first attempt, a fairly straightforward leastsquare linear fit to the layer-centroids is applied the BGO image. To minimize the effect of some "irregular" backs plash and/or secondary particles, layers with energy deposite smaller than 3\% of the total energy deposition are discarded. For the remained layers with sufficient fraction of energy deposition, the centroid of the energy deposition of this layer is calculated as:

$$
C_{l}=\sum X_{i} E_{i} / \sum E_{i}
$$

where $X_{i}$ and $E_{i}$ is the position and the energy deposition of the ith BGO bar of the layer, and the summation is over all the $22 \mathrm{BGO}$ bars of a layer. In case at least three effective layer centroids are available, a weighted least-square linear fit is applied to the position of the layer centroids to find an estimation of the shower axis, with each position being weighted by the total energy deposit 
of the layer. This simple algorithm turns out to be very robust and is able to reconstruct a BGO track, good or poor, for more than $98 \%$ of all the events. The limitations of this method, however, evidence themselves with accumulating of flight data and simulation data. Firstly, there is a very obvious "coarse grain effect" due to the relative large dimension of each BGO bar. For example, an event slightly (a few degrees) deviate from a right angle incidence still leaves a straight row of layer maximums in the BGO that dominate the layer centroid position given the considerable size of each bars, resulting into a reconstructed BGO track very close to a right angle incidence. The "grid" structure shown in Fig. 3 is a result of this "coarse grain effect", i.e., to pile up tracks at certain angles due to the discrete measurement of the positions. Also, this algorithm is found to provide a systematically smaller incident angle, especially for the events of large incident angle. This is due to the limitation of the least-square linear fit, which intends to avoid a fitting result of large inclination, given the mechanism of this method is to minimize the square of the $\Delta Y^{2}=\left(Y_{\text {fit }}-Y_{\text {measure }}\right)^{2}$.
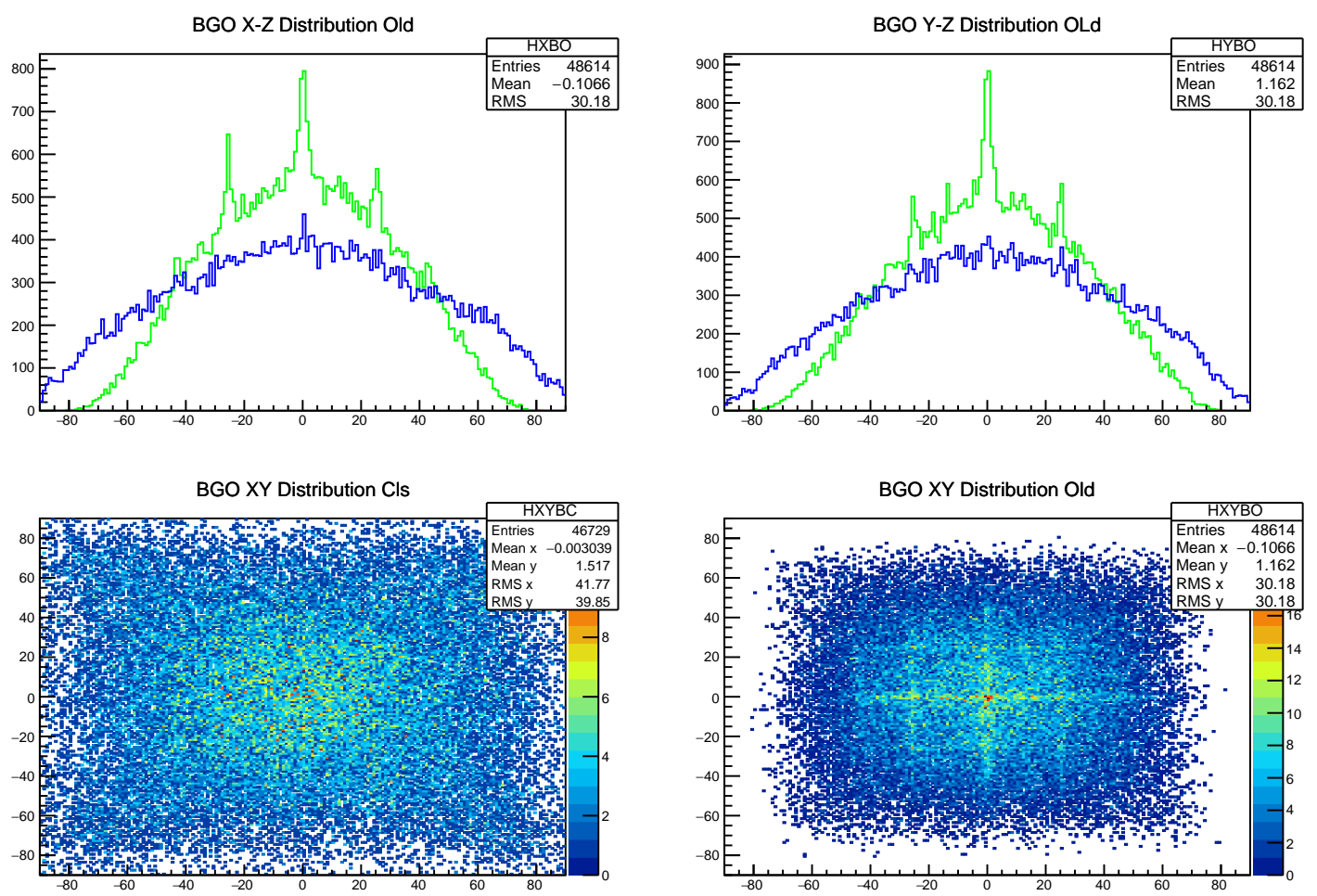

Figure 2: Distributions of the incident angle of the reconstructed BGO tracks for flight data. One dimensional distributions of the incident angle of the BGO tracks reconstructed using the old (green histograms) and new (blue histograms) algorithm are shown for the X-Z (top-left panel) and Y-Z (top-right panel) plane, respectively. Two dimensional distributions of the incident angle resulted from the same data are also shown for the new (bottom-left) and old (bottom-right) method. Obvious "coarse grain effect" seen in the results obtained by the old method as the peaks in the one dimensional distribution and the grids in the two dimensional ones is but greatly mitigated by the new method. Also, a great number of events with large incident angle are mistakenly reconstructed to be events with small incident angle by the old method. See text for more details. 


\section{BGO track reconstruction: improvement}

With the accumulation of massive fight data as well as simulation data during the smooth running of the entire DAMPE project, significant improvements have been made in almost all aspects of detector calibrations and event reconstructions based on our better understanding of the DAMPE detector performance. An improved BGO track reconstruction algorithm has also been proposed.

The new BGO track reconstruction procedure also starts with layer selection. A 3\% energy deposition fraction threshold is still applied to discard layers without sufficient energy deposition, but only for the layers after the shower maximum layer that has the largest energy deposition. All the layers before the shower maximum are preserved for track reconstruction, as we find that the BGO bars fired before the shower maximum, although may account only a small fraction of total energy deposition, provide useful, some times accurate information of the incident particle. Including the fired BGO bars with small fraction of energy deposition after the shower maximum in the track reconstruction is but found to reduce the quality of the result.

Then a key procedure of the new method is to search for the "clusters" of fired bars in each BGO layer. A cluster is built starting from the bar with the maximum energy deposit and associating to it all the neighboring bars on both sides with decreasing energy deposits. The cluster construction is terminated when one of the following conditions is met: (1) the side of BGO is reached; (2) a non-fired bar is found; (3) a bar with increasing energy deposit is found. Finally, we make clusters symmetric about the maximum energy bar. Therefore, if the left (right) tail of the fired bar cluster has more bars than the right (left) tail, the bars in excess are removed. We then set the energy of all the bars found for the first cluster to be zero and search for the second cluster of the layer. The procedure is repeated until all the fired bars in a layer are exhausted. We but allow one cluster per layer at most by choosing the cluster with the largest total energy deposition (not necessary to be the cluster associated with the maximum energy bar). Instead of using the centroid of each cluster for a fitting as being in the old algorithm, the new procedure performs a weighted linear fit to the positions of all the bars in the clusters counted, with each bar being weighted with the corresponding energy deposit. The fitting result, however, is found to bear some systematic bias for inclined incident particles. To minimize this bias, we rotate the coordinate to align the $X$ axis with the track direction obtained from the first fit. A second fit is then performed in the new coordinate system. A twice repeating of this procedure is found to be sufficient to make the result converge and the final result is obtained by converting back into the original coordinate system. The direction found by the BGO track reconstruction (if available) is used as a seed for the STK track reconstruction.

\section{Summary}

The reconstructed shower axis in the BGO detector that provides a good estimation of the incident particle direction within the uncertainty of a few degrees is a very good complement to the STK track especially for the events of very high energy. Our first attempt of BGO track reconstruction based on a linear fit to the energy deposition centroids of each BGO layer but gives relatively large statistical and systematic errors. An improved BGO track reconstruction algorithm based on 

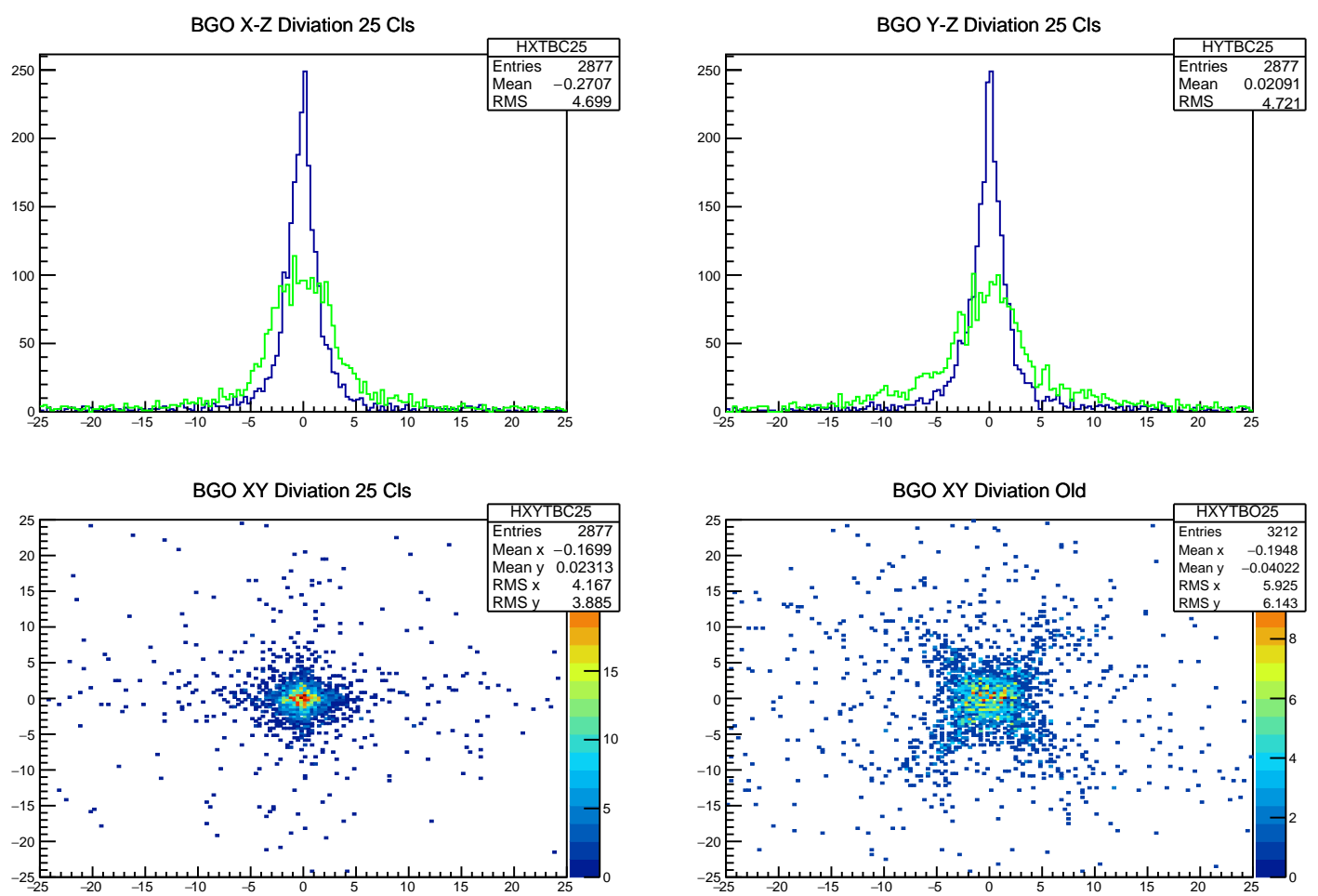

Figure 3: Distribution of the error in the incident angle of the reconstructed BGO tracks for simulation data of $600 \mathrm{GeV}$ electrons. Same as Fig. 2., the two top panels show the one dimensional distributions of the errors for the $\mathrm{X}-\mathrm{Z}$ (top-left panel) and Y-Z (panel) plane reconstruction results obtained by the old (green histogram) and new (blue histogram) method. The two dimensional distributions are also shown for the results of the old (bottom-right) and new (bottom-left) method

cluster searching has been proposed. Preliminary tests using both flight data and simulation data show that the new algorithm provides results of higher accuracy without losing efficiency. A journal paper dedicated the BGO track reconstruction under preparation will provide more details of the algorithm as well as thorough test results.

\section{Acknowledgement}

We appreciate the very helpful comments from a number of colleagues in developing the BGO track reconstruction algorithm. We thank Tie-Kuang Dong and Chuan Yue for testing the new algorithm for charge measurement and proton selection.

The DAMPE mission is funded by the strategic priority science and technology projects in space science of Chinese Academy of Sciences. This work is supported by the Astronomy Joint Grant U1631111.

\section{References}

[1] J. Chang, Dark Matter Particle Explorer: The First Chinese Cosmic Ray and Hard gamma-ray Detector in Space, Chin. J. Spac. Sci., 34550 (2014). 
[2] J. Chang et al. [DAMPE collaboration], The dark matter particle explorer mission, submitted to Asropart. Phys., (arXiv:1706.08453) (2017).

[3] K. K. Duan, et al., PoS (ICRC2017), 775 (2017).

[4] Y. Z. Fan, B. Zhang, \& J. Chang, Electron/positron Excesses in the Cosmic Ray Spectrum and Possible Interpretations, Int. J. Mod. Phys. D, 192011 (2010).

[5] J. L. Feng, Dark Matter Candidates from Particle Physics and Methods of Detection, Annu. Rev. Astron. Astrophys. 48, 495 (2010).

[6] Y. F. Liang, et al., Search for a gamma-ray line feature from a group of nearby galaxy clusters with Fermi LAT Pass 8 data, Phys. Rev. D, 93 (2016) 103525.

[7] M. Munoze et al., Anisotropy searches with DAMPE (PoS(ICRC2019)) (2019).

[8] T. K. Dong, et al., Astropart. Phys. 105 (2019) 31.

[9] X. Wu et al., Proceedings of the 34th International Cosmic Ray Conference (PoS(ICRC2015)) (2015) 1192.

[10] Z. L. Xu, et al., Res. Astron. Astrophys. 18 (2018) 27. 